\title{
Corela
}

Cognition, représentation, langage

7-1 | 2009

Vol. $7, \mathrm{n}^{\circ} 1$

\section{Les nasales en ikwere}

\section{Sylvester Osu et G. N. Clements}

\section{OpenEdition}

\section{Journals}

Édition électronique

URL : http://journals.openedition.org/corela/134

DOI : 10.4000/corela.134

ISSN : 1638-573X

\section{Éditeur}

Cercle linguistique du Centre et de l'Ouest - CerLICO

\section{Référence électronique}

Sylvester Osu et G. N. Clements, «Les nasales en ikwere », Corela [En ligne], 7-1 | 2009, mis en ligne le 06 septembre 2010, consulté le 19 avril 2019. URL : http://journals.openedition.org/corela/134 ; DOI : $10.4000 /$ corela.134

Ce document a été généré automatiquement le 19 avril 2019

\section{(c) (i) (2)(2)}

Corela - cognition, représentation, langage est mis à disposition selon les termes de la licence Creative Commons Attribution - Pas d'Utilisation Commerciale - Partage dans les Mêmes Conditions 4.0 International. 


\title{
Les nasales en ikwere
}

\author{
Sylvester Osu et G. N. Clements
}

\section{NOTE DE L'ÉDITEUR}

Attention : pour un affichage correct de certains caractères, télécharger et installer la police "Doulos SIL" (police unicode UTF-8) : http://scripts.sil.org/cms/scripts/page.php? site_id=nrsi\&item_id=DoulosSIL_download\#1fd0063a

\section{Introduction}

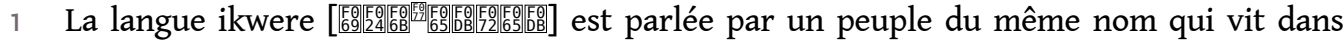
l'Etat des Rivières (Rivers State) dans une vaste région située au sud-est du Nigéria comprenant des villes telles que Rumudioga, Elele-Alimini à l'ouest, Omagwna et Igwuruta à l'est, en passant par Ogbakiri, Emowha et Isiokpo. Elle est également parlée dans la région d'Obio ainsi qu'à Port Harcourt, capitale de l'Etat des Rivières. Ce travail

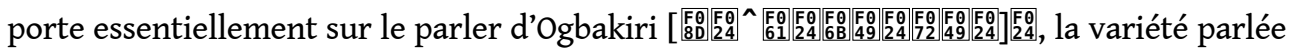
par le premier auteur (SO). La langue ikwere est une langue igboïde du groupe BenueCongo, de la famille Niger-Congo (Williamson \& Blench 2000), qui compte environ 980, 000 locuteurs ${ }^{1}$.

2 Il existe très peu de travaux en linguistique sur l'ikwere, et ceux qui existent concernent rarement la phonologie de cette langue. Cependant, on trouve ici et là quelques passages évoquant la phonologie dans des travaux qui portent principalement sur d'autres sujets. Parmi ces travaux figure en bonne place le manuel scolaire destiné aux enseignants de l'école primaire édité par Kay Williamson en 1970. Williamson y fait une présentation de l'orthographe officielle de l'ikwere accompagnée de commentaires sur son système phonologique. Ce manuel est actuellement en cours de révision. Dans un article sur la nasalisation dans les langues kwa, Williamson (1973) fait également mention de l'ikwere. Worukwo (1983) lui, présente quelques rudiments du système tonal de l'ikwere avec une 
brève discussion qui porte sur sa phonologie segmentale. Donwa-Ifode \& Ekwulo (1987) présente une version plus récente de l'orthographe de l'ikwere et comprend également une analyse phonémique proche de celle de Worukwo précitée. Enfin, Osu (1998), dans une perspective énonciative, étudie les particules verbales de l'ikwere du point de vue de leur fonctionnement dans la langue. Ce dernier travail contient également quelques renseignements sur la morphophonologie de l'ikwere.

Le but principal de cet article est de montrer que la nasalité dans la langue ikwere est distinctive pour les voyelles mais pas pour les consonnes. Cette étude s'inscrit dans un programme de recherche plus large que nous avons entrepris sur la phonologie de l'ikwere. Dans une précédente publication (Clements \& Osu 2002), nous nous sommes intéressés à des aspects phonétiques des deux consonnes représentées par $k p$ et $g b$ dans l'orthographe. Nous y avons montré que ces consonnes, que nous transcrivons avec les symboles phonétiques ^et 医^, ne sont ni des labio-vélaires, ni des obstruantes, ni des injectives, mais des occlusives labiales non-explosives, légerement labialisées et probablement accompagnées d'une légère vélarisation. L'on verra dans a discussion qui suit qu'elles se comportent bel et bien comme d'autres consonnes non-obstruantes du point de vue de la nasalisation consonantique ${ }^{2}$.

\section{L'ikwere: une langue sans consonnes nasales distinctives}

Dans cette section, nous présentons d'abord les voyelles et les consonnes dans le contexte des syllabes CV. Les groupes NC (nasale + consonne) sont ensuite examinés afin de montrer que l'élément nasal n'est pas une consonne mais une vocoïde nasale qui n'a pas de lieu d'articulation propre.

\subsection{Les voyelles orales et nasales}

5 L'ikwere est une langue avec une structure syllabique ouverte, ce qui implique que toutes les syllabes sont d'une forme de base $\mathrm{CV}$, avec la position d'attaque $\mathrm{C}$ obligatoire.Nous montrerons que les exceptions à cette règle ne le sont qu'en apparence ${ }^{3}$.

6 Les paires minimales ou quasi-minimales en (1) ci-dessous montrent que la nasalité est distinctive pour les voyelles.

\begin{tabular}{|c|c|c|}
\hline (1) & orale & nasale \\
\hline & 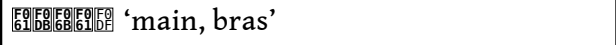 & 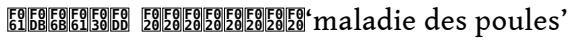 \\
\hline & 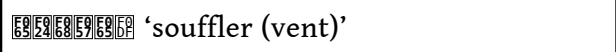 & 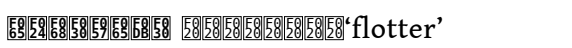 \\
\hline & 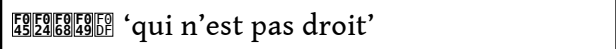 & 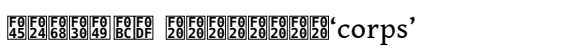 \\
\hline & 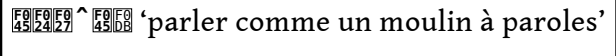 & 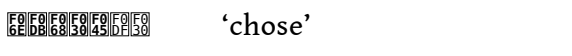 \\
\hline & 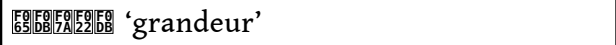 & 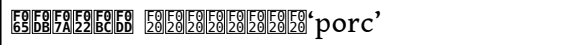 \\
\hline & 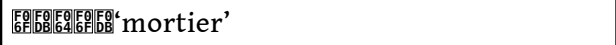 & 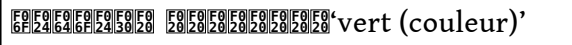 \\
\hline
\end{tabular}




\begin{tabular}{|c|c|}
\hline 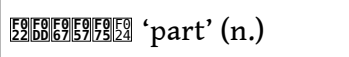 & i䜿 \\
\hline 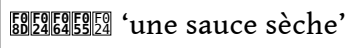 & 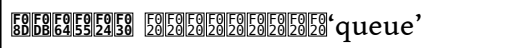 \\
\hline 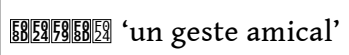 & 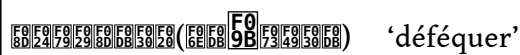 \\
\hline
\end{tabular}

$7 \quad$ Il ressort de ce contraste le système vocalique présenté en (2).

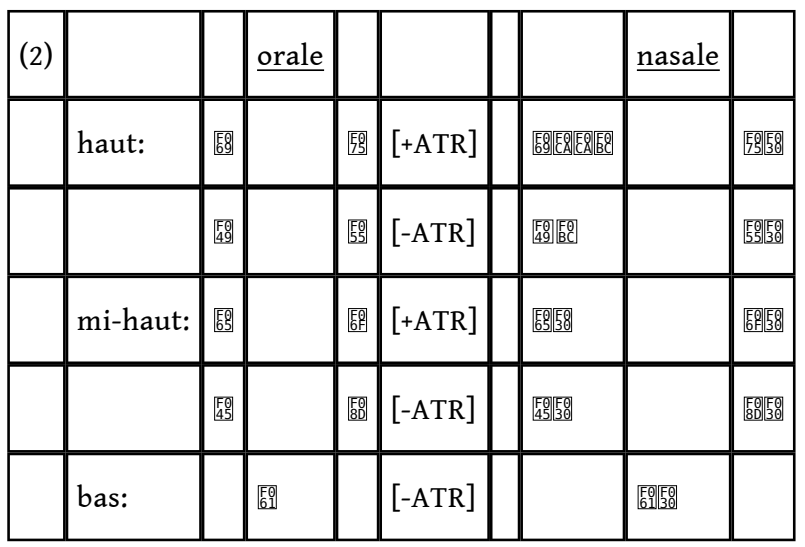

8 Dans la langue igbo, langue très proche de l'ikwere, la voyelle / $/$ Fi $_{5} /$ n'a pas un statut

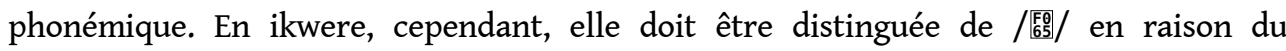

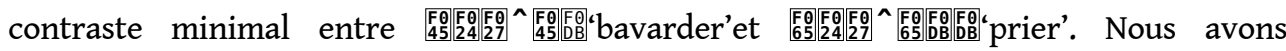

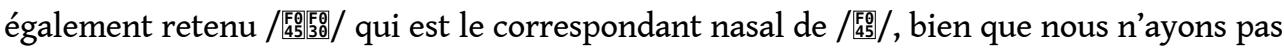

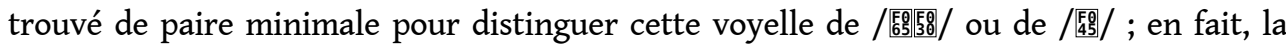
majorité des occurrences de / $/ 450$ [50/ dans notre corpus sont dues soit à l'harmonie vocalique par rapport au trait ATR, soit à l'harmonie nasale (pour cette dernière voir Clements et Osu 2003, 2005). Cela dit, on peut trouver cette voyelle dans un nombre limité

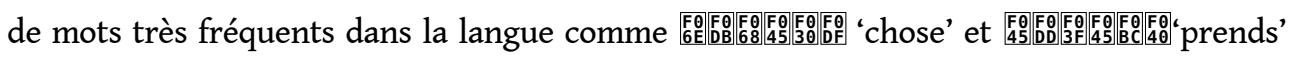
où elle pourrait difficilement être dérivée d'une autre voyelle.

9 On note un certain nombre de restrictions relatives à la distribution des voyelles orales et nasales. Premièrement, s'il est vrai que les voyelles orales et nasales s'opposent derrière une consonne orale, seules les voyelles nasales peuvent apparaître derrière une consonne nasale. Nous revenons sur ce point dans la section suivante.Deuxièmement, les voyelles nasales en (2) n'apparaissent jamais en position initiale de mot. Nous verrons dans la section 2.5 qu'unenasale moraïque sans lieu d'articulation spécifié apparaît dans cette position initiale de motoù elle s'oppose aux voyelles orales en (2).

10 Le système vocalique de l'ikwere est caractérisé par deux sortes d'harmonie. D'abord, comme beaucoup de langues de l'Afrique de l'Ouest, l'ikwere a un système d'harmonie vocalique selon lequel toutes les voyelles d'un mot, sauf quelques exceptions (très systématiques d'ailleurs), sont produites avec la racine de la langue avancée ([+ATR]) ou non avancée ([-ATR]). Un tel système engendre des alternances assez productives comme

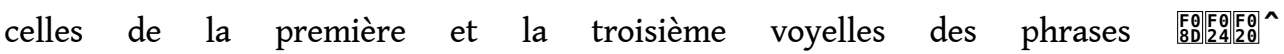

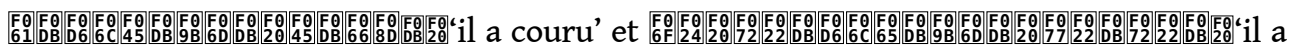
mangé'. Il convient de signaler que l'harmonie ATR est très fréquente dans les exemples 
que nous discuterons plus loin, même si pour ne pas alourdir cet article nous avons choisi de ne pas les commenter au fur et à mesure.

11 L'ikwere a aussi un système d'harmonie "horizontale » selon lequel les voyelles non bassesde la même hauteur sont soit antérieures, soit postérieures. Cela signifie que les séquences telles que $\mathrm{CiCu}, \mathrm{CuCi}, \mathrm{CeCo}$, et CoCe ainsi que leurs correspondantes ([-ATR]) sont exclues. Par conséquent, les voyelles qui se suivent dans un mot sont soit identiques,

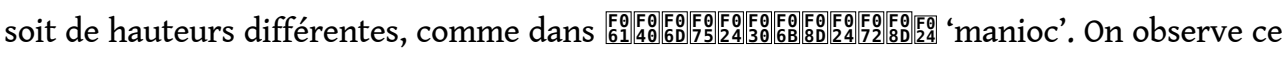
type d'harmonie dans un domaine que nous proposons d'appeler la racine phonologique. Ce domaine comprend tout élément dans une racine (morphologique) sauf les syllabes $\mathbf{V}$ et rV à l'initiale des noms. La plupart des exceptions à l'harmonie horizontale comportent

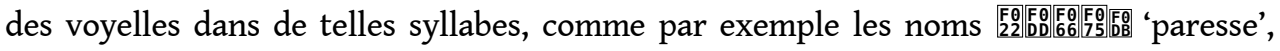
每 d'autres exceptions dans des idéophones tels que [F] exprime l'idée d'un noir très foncé).

\subsection{Les consonnes orales et nasales en surface}

Les consonnes en surface (3) appartiennent à trois séries qui apparaissent aussi bien à l'initiale du mot qu'en position intervocalique. Les deux premières regroupent les consonnes orales tandis que la troisième contient les nasales. Les symboles en (3) ont leur

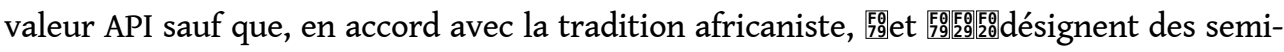
voyelles palatales et $\mathbf{c}$ et $\mathbf{j}$ désignent des affriquées palato-alvéolaires. En plus, à défaut de symboles API, ^et [27 ${ }^{\wedge}$ désignent les occlusives non-explosives. De façon générale, $\mathbf{r}$ représente une vibrante battue, mais ce son est parfois réalisé comme une approximante. Quant à $\underset{\mathrm{Ca}}{\mathrm{E}}$, elle est toujours approximante.

(3) Série A: obstruantes

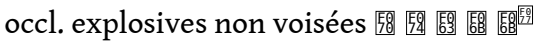

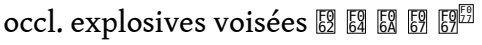

fricatives non voisées 楷區

fricatives voisées 5 殹

Série B: non-obstruantes orales

non-explosives voisées ^

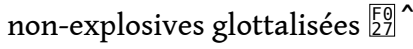

approximantes latérales

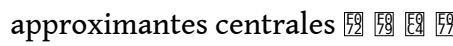

aspirées 熙 熙[5]

Série C: non-obstruantes nasales

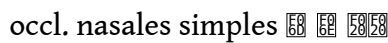

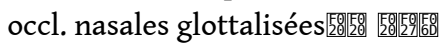

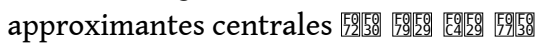

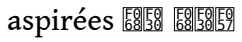

13 Les trois regroupements en (3) s'appuient sur des critères à la fois distributionnels et phonétiques. Les consonnes de la série A sont toutes des obstruantes et apparaissent devant des voyelles orales et nasales. Les consonnes de la série B sont des nonobstruantes et n'apparaissent que devant des voyelles orales. Les consonnes de la série $C$ enfin, ne sont pas non plus obstruantes et elles apparaissent uniquement devant des voyelles nasales. Les consonnes de type B et $\mathrm{C}$ sont en distribution complémentaire ${ }^{5}$. Nous proposerons ci-dessous qu'elles ne constituent qu'une seule série au niveau sousjacent. 


\subsection{Remarques sur les réalisations phonétiques} auteur enregistrées au Laboratoire de Phonétique de l'Université de Paris 3. Des petits corpus ont été construits pour permettre d'approfondir certains points, et ils consistent en plusieurs répétitions d'ensembles de mots produits en forme de citation ainsi que de courtes phrases. Nos observations acoustiques elles, sont fondées sur des spectrogrammes (non reproduits ici) que nous avons obtenus à l'aide du logiciel CSRE (Computerized Speech Research Environment), v. 4.2, de AVAAZ Innovations (Canada). Quant à l'étude articulatoire, nous avons mesuré le débit d'air nasal à l'aide du système d'acquisition de données PCQuirer (SciCon, Los Angeles) ; voir plus de détail ci-dessous.

\subsubsection{Les obstruantes devant des voyelles nasales}

obstruantes de la série A restent pour la plupart orales devant une voyelle nasale, mais certaines ont des variantes créées par un léger décalage entre le relâchement de la consonne et l'abaissement du voile du palais. En ce qui concerne les occlusives non voisées, les spectrogrammes et les enregistrements du débit d'air indiquent qu'elles peuvent être suivies d'une aspiration plus ou moins importante ; ainsi, par exemple, la durée du relâchement aspiré de /t/ est-elle presque doublée devant une voyelle nasale. Les occlusives sonores sont parfois réalisées avec une post-nasalisation dans ce contexte :

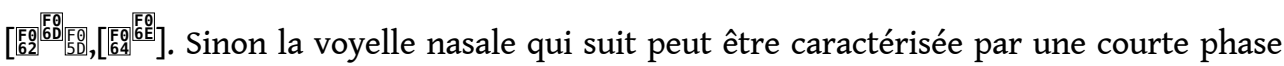
orale au début.

Aussi, la réalisation de la fricative [f] devant une voyelle nasale s'accompagne d'une postnasalisation qui est caractérisée par un débit d'air nasal non voisé.

\subsubsection{Les occlusives non-explosives ^et $\mathrm{E}_{27}^{\left[{ }^{\natural}\right.} \wedge$}

17 A l'inverse des consonnes explosives $\mathbf{b}$ et $\mathbf{p}$ de la série $\mathrm{A}$, les non-explosives ^ ${ }^{\text {et }}{ }_{27}^{\mathrm{E}}{ }^{\wedge}$ de la série $B$ n'impliquent ni une augmentation de la pression de l'air pendant leur tenue, ni une explosion audible au moment de leur relâchement. C'est pourquoi elles sont classées en (3) comme des non-obstruantes, le terme "obstruant » renvoyant à des sons qui se caractérisent par une augmentation de la pression à l'intérieur de la cavité vocale (voir par ex. Stevens 1983). Historiquement, ces consonnes sont desvestiges des labialesvélairesgb et kp que l'on trouve encore dans d'autres langues igboïdes (Williamson 2000) et peuvent être apparentées aux injectives de certains parlers igbo (Ladefoged et al. 1976). Une étude phonétique des non-obstruantes de l'ikwere(Clements \& Osu 2002) révèle cependant qu'aucune des deux n'implique une occlusion vélaire, et que dans la mesure où ni l'une ni l'autre ne mettent un jeu un mécanisme glottique de réglage de débit d'air, elles ne sont pas non plus des injectives au sens traditionnel du terme.

\subsection{Les consonnes orales et nasales non-obstruantes : analyse phonémique}

La distribution complémentaire des consonnes de la série $\mathrm{B}$ et de la série $\mathrm{C}$ se confirme dans toutes les positions. ${ }^{6}$ Par conséquent on peut les dériver d'une seule série sous- 
jacente non spécifiée pour le trait de nasalité. Les consonnes de la série en question sont réalisées orales devant une voyelle orale (série B) mais nasales devant une voyelle nasale (série C).

19 En (4) ci-dessous, les consonnes de type $B$ et $C$ sont regroupées en fonction du phonème auquel elles correspondent.

\begin{tabular}{|c|c|c|c|c|c|c|c|}
\hline \multirow[t]{2}{*}{ (4) } & \multicolumn{4}{|c|}{ devant une voyelle orale } & \multicolumn{3}{|c|}{ devant une voyelle nasale } \\
\hline & 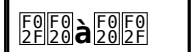 & 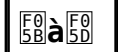 & 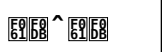 & 'peinture' & 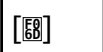 & 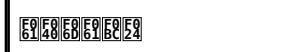 & 'machette' \\
\hline & 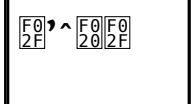 & {$\left[{ }_{50}, n\right]$} & 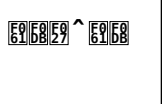 & 'camaraderie' & 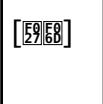 & 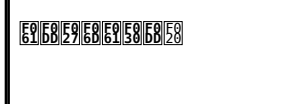 & $\begin{array}{l}\text { 'chemin, } \\
\text { route' }\end{array}$ \\
\hline & 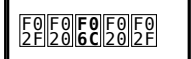 & 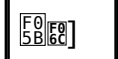 & 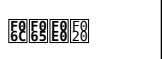 & 'comme' & 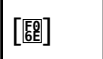 & 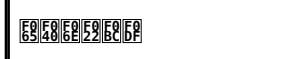 & 'éléphant' \\
\hline & 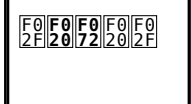 & 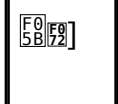 & 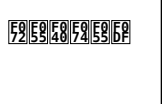 & \begin{tabular}{|l} 
'batonnet frotte- \\
dent'
\end{tabular} & 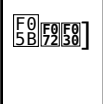 & 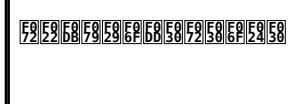 & $\begin{array}{l}\text { 'charbon } \\
\text { de bois' }\end{array}$ \\
\hline & 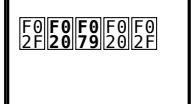 & 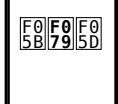 & 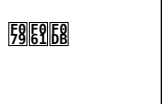 & $\begin{array}{l}\text { 'il/elle' } \\
\text { (emphatique) }\end{array}$ & [[50F[2] & 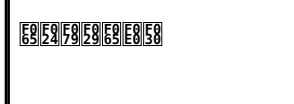 & 'donner' \\
\hline & 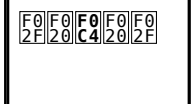 & 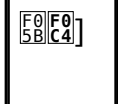 & 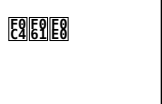 & 'laisse-le!' & 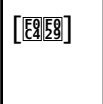 & 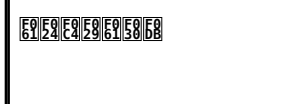 & $\begin{array}{l}\text { 'se dorer } \\
\text { au soleil' }\end{array}$ \\
\hline & 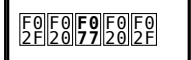 & \begin{tabular}{|l|l|}
$50][7][50$ \\
58
\end{tabular} & 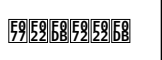 & 'nourriture' & [59558] & 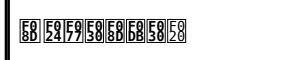 & 'boire' \\
\hline & 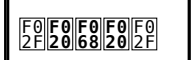 & 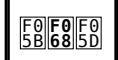 & 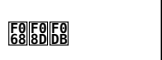 & 'dit' & 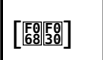 & 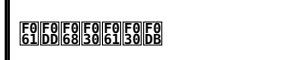 & 'suffire' \\
\hline & 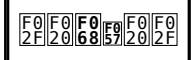 & 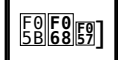 & 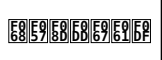 & |'sors!' & 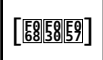 & 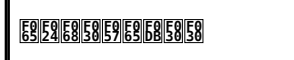 & 'flotter' \\
\hline
\end{tabular}

20 Devant une voyelle nasale, la nasalisation s'étend sur toute la durée de ces consonnes,

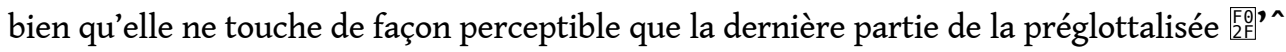
[F⿱ de l'occlusion glottale.

21 Les paires de consonnes en (4) partagent la plupart de leurs traits : pour l'essentiel, les consonnes de la colonne de droite ne se distinguent de leurs correspondantes de la colonne de gauche que par le fait qu'elles ont le trait [+nasal] en plus ${ }^{7}$. On remarque aussi des alternances régulières entre certaines de ces paires; comme illustrée en (5) par

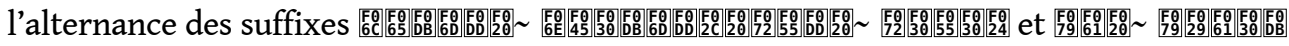

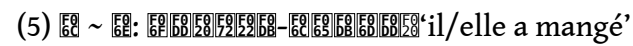

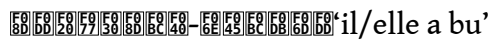

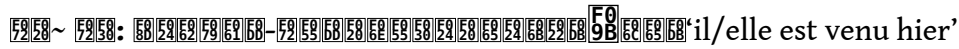

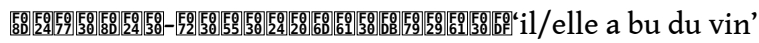

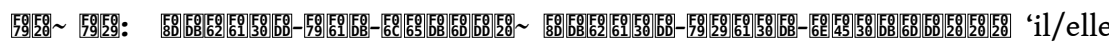
est entré' (variantes libres)

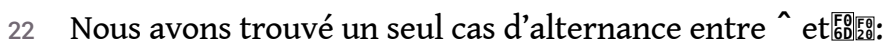

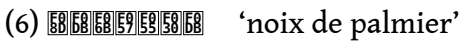




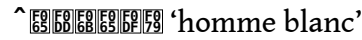

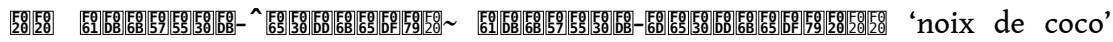 (variantes individuelles)} arguments permettent de soutenir l'idée que ce son est bien une vocoïde, c'est-à-dire un segment non consonantique, au niveau sous-jacent. D'abord, il apparaît devant une consonne, une position occupée ailleurs uniquement par des voyelles. Ensuite, comme toute autre voyelle, il est susceptible de porter un ton. Puis, nous avons signalé en note 3 qu'un coup de glotte non phonémique se manifeste devant une voyelle en début d'énoncé. Or, ce coup de glotte se manifeste également devant /原㵧/ en début d'énoncé alors qu'il n'apparaît pas devant des consonnes, même lorsqu'il s'agit des nasales. Considérons quelques exemples de cette réalisation en (8) :

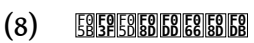

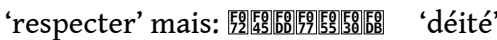

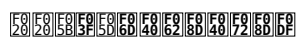

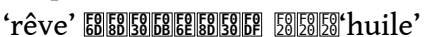

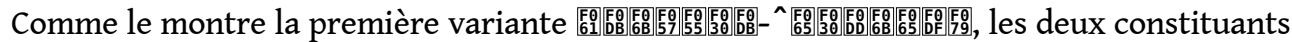
composé 'noix de coco' existent dans la langue indépendamment l'un de l'autre. 政 voyelle finale du premier constituant du mot à la première syllabe du second, et ainsi le ^ s'est transformé en $\mathbf{m}$.

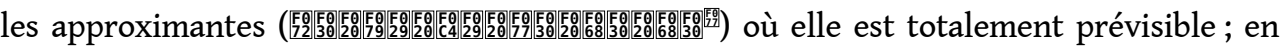
$/, /{ }^{\wedge} /$ et / $/ \mathbb{E C}_{0} /$ respectivement.

\subsection{La nasale moraïque} qu'aucune voyelle nasale présentée en (2) n'apparaisse à l'initiale du mot, on peut voir au

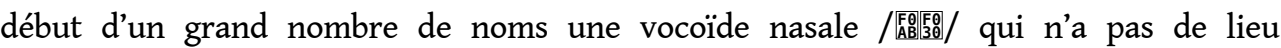
d'articulation propre mais qui est susceptible de porter un ton. Par convention nous

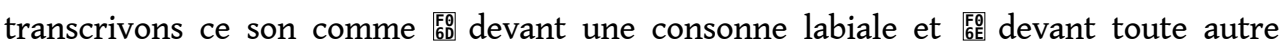

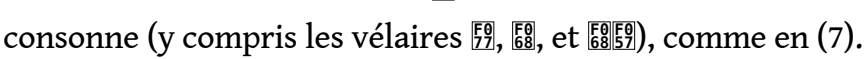

\begin{tabular}{|c|c|c|c|c|}
\hline (7) & 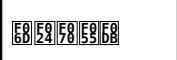 & 'pou des sables' & 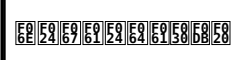 & 'chaise' \\
\hline & 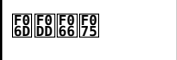 & 'corne' & 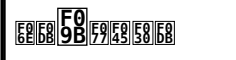 & (logophorique) \\
\hline & 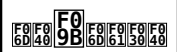 & 'beauté' & 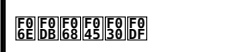 & 'chose' \\
\hline & 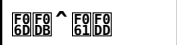 & 'palourde' & 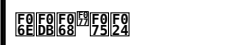 & 'perte' \\
\hline
\end{tabular}

28 Tout ceci montre que les nasales devant une consonne sont plutôt des vocoïdes et non des consonnes. 

phonèmes consonantiques dont la réalisation principale est nasale) a déjà fait l'objet de discussion. Dans un article bien connu, Ferguson (1963) a postulé l'existence d'au moins une consonne nasale primaire dans toutes les langues. Mais Ferguson lui-même avait reconnu quelques années plus tard qu'il existe une poignée de langues dans lesquelles on ne trouve pas une telle consonne. Une de ces langues est le lushootseed (ou salishan de Puget Sound), dans laquelle, selon Ferguson (1975: 176) «la nasalité semble ne pas fonctionner du tout » même au niveau phonétique.

D'autres exemples ont été trouvés depuis, notamment dans des langues dans lesquelles les consonnes nasales ne sont que des réalisations prévisibles des consonnes orales en contexte nasal, comme en ikwere. De telles langues se trouvent particulièrement (mais pas exclusivement) dans une zone d'Afrique de l'Ouest allant du Libéria jusqu'au sud du Nigeria (pour des exemples voir, entre autres, celles citées dans Schachter \& Fromkin 1968, Le Saout 1973, Bentinck 1975, Capo 1981, 1991, Bole-Richard 1983a, b, 1984, Ihionu 1984, et Creissels 1994). On les trouve également en Amérique du Sud. La liste en (10), qui n'est évidemment pas exhaustive, présente à la fois des langues citées par des auteurs précités et d'autres prises dans Bole-Richard (1985), Maddieson (1984), et Cohn (1993a, b).

(10) Langues sans consonnes nasales primaires

Amérique du Nord: hidatsa, lushootseed, quileute

Amérique du Sud: barasano, tucano, epena pedee, cubeo, andoke, pirahã

Pacifique: rotokas

Afrique de l'Ouest (Niger-Congo):

Mandé: kpelle, dan, gouro, yaouré, wan, mwan, gban, toura

Gur: bwamu, senadi/senoufo 
Kru: nyabwa, w

Kwa: éwé, fon, akan, ébrié, avikam, abouré, abron

Igboïde: igbo de mbaise, ikwere

Oubangui: yakoma de voyelles nasales, les chercheurs ont quelquefois observé que les voyelles nasales semblent faiblement nasalisées, voire pas nasalisées du tout, après les consonnes nasales (voir Moñino (1995), Stewart (1999) pour une discussion sur ce point dans la langue gbaya). Au début de notre travail sur l'ikwere, le deuxième auteur, d'origine anglophone, avait une difficulté semblable à percevoir la nasalité des voyelles nasales ikwere lorsque celles-ci suivent des consonnes nasales. On peut cependant, se poser la question suivante : est-ce que ces perceptions correspondent à une réalité phonétique, ou sont-elles attribuables a l'influence de la langue maternelle de l'observateur ? Une étude de Beddor et al. (2001) montre que dans un contexte expérimental, les sujets anglophones ont plus de difficulté à percevoir la nasalisation d'une voyelle nasalisée lorsque, toutes choses étant égales par ailleurs, la voyelle suit une consonne nasale.

Pour tenter de répondre à cette question, nous avons enregistré et numérisé un ensemble de mots dans lesquels se trouvent, entre autres consonnes, les nasales $\mathbf{m}$ et $\mathbf{n}$ suivies de différentes voyelles. Après avoir obtenu un spectrogramme de chaque mot, nous avons sélectionné et écouté les voyelles afin de bien les évaluer auditivement. Dans tous les cas, les voyelles suivant $\mathbf{m}$ et $\mathbf{n}$, une fois isolées de leur contexte, nous ont semblé aussi fortement nasalisées que toute voyelle nasale suivant une consonne orale. Ainsi, par 


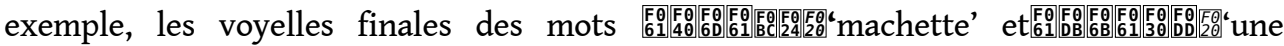
maladie des poules' nous ont paru autant nasalisées l'une que l'autre lorsque nous les avons extraites de leurs contextes.

D'autre part, nous avons mesuré le débit d'air nasal dans la production d'un corpus de plusieurs dizaines de mots et de phrases ikwere à l'aide du logiciel PCQuirer (voir cidessus). Des masques en caoutchouc étanches à l'air ont été fixés au nez et à la bouche du premier auteur (SO).Le débit d'air nasal a été mesuré au moyen d'un transducteur de pression qui était attaché à un tube inséré dans un masque nasal,et le débit d'air oral a été simultanément mesuré au moyen d'un autre tube inséré dans un masque oral. Le signal audio a été enregistré en même temps. A la suite de chaque enregistrement, le signal audio ainsi que les deux tracés du débit d'air ont été alignés sur un écran d'ordinateur,

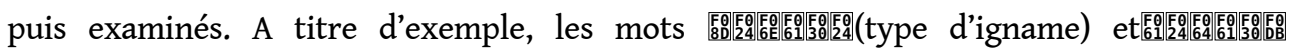
'premier enfant de sexe féminin', extrait d'un même enregistrement, sont présentés en Figure 1.

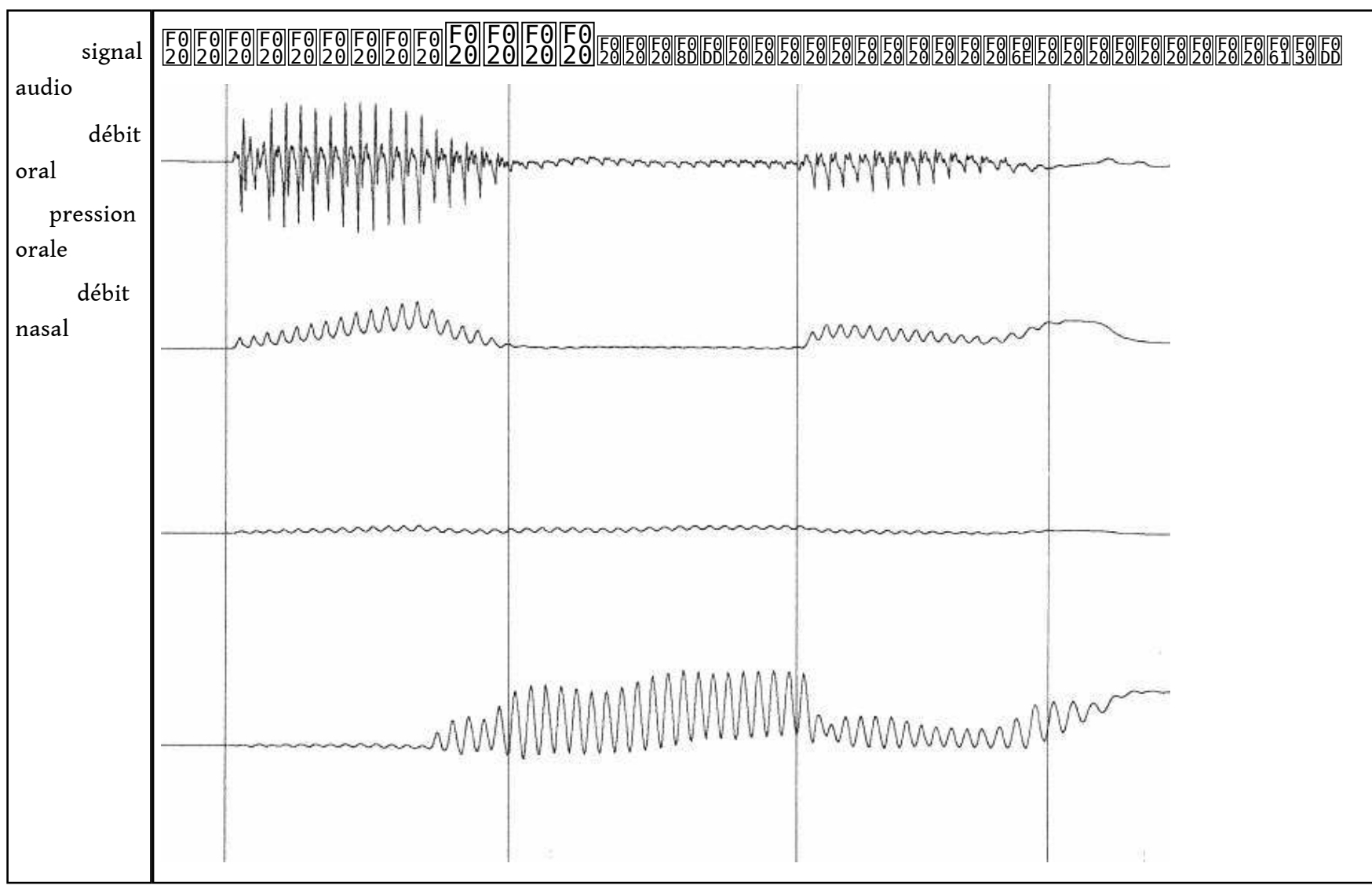




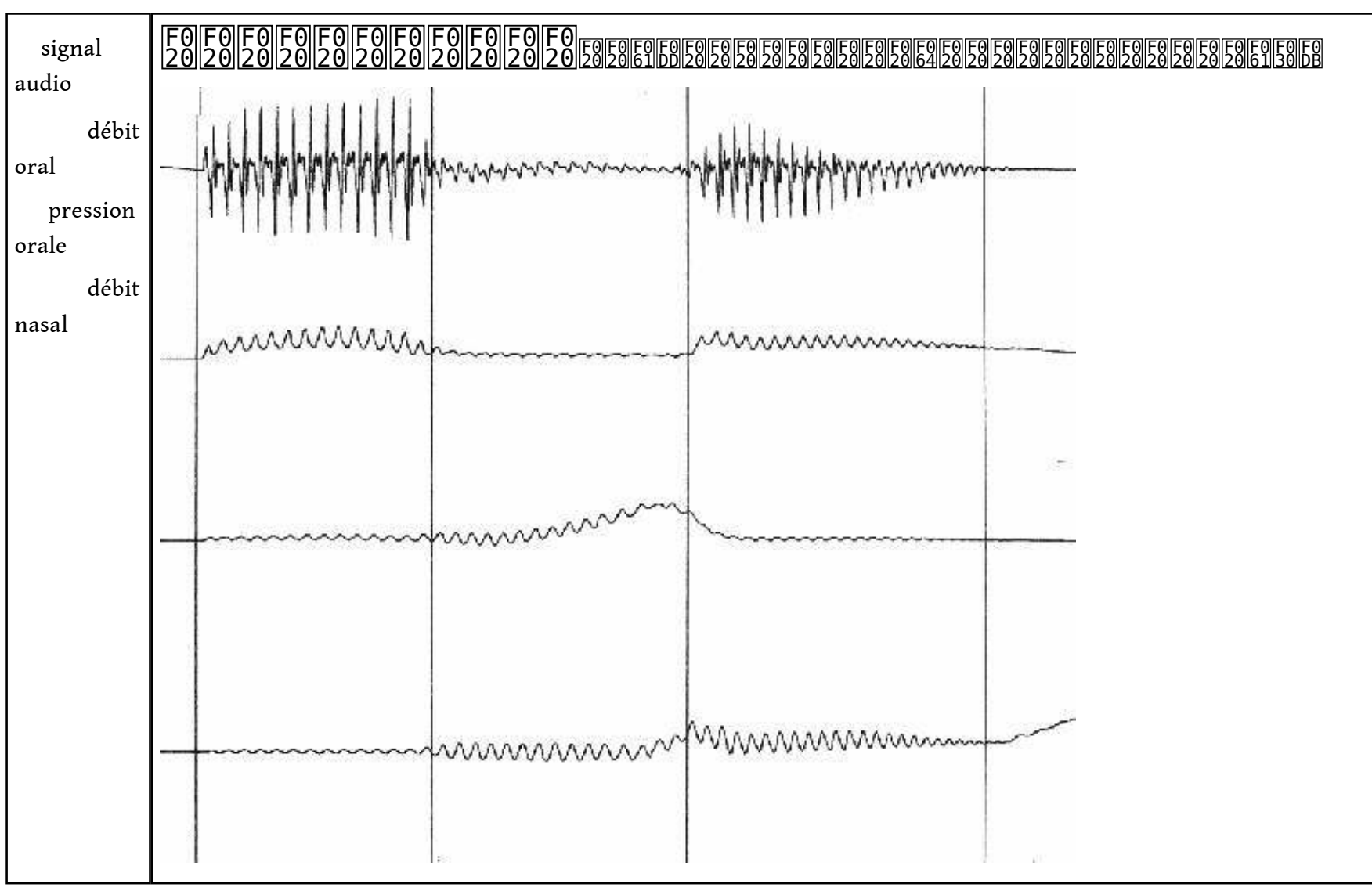

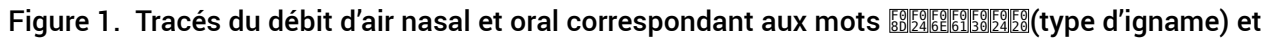

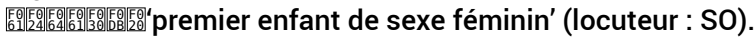

Comme on peut le constater, la seconde voyelle de chaque mot présente un débit d'air nasal conséquent, alors que la première présente un débit nasal assez négligeable sinon inexistant. On peut noter en particulier que dans ces tracés, la voyelle suivant $\mathbf{n}$ présente un débit d'air nasal aussi important que celle qui suit $\mathbf{d}$.

41 Ces exemples, qui sont représentatifs de tous ceux que nous avons examinés, montrent que les voyelles qui suivent les consonnes nasales en ikwere sont aussi nasalisées du point de vue phonétique que les voyelles nasalesqui suivent les consonnes orales. Rien n'empêche donc de considérer la nasalisation comme phonologique dans les deux cas.

\section{Les autres consonnes nasales}

Dans cette section, nous considérons les consonnes nasales qui a priori, ne se laissent pas analyser de la même façon que celles que nous avons examinées jusqu'ici. L'analyse porte d'abord sur le préfixe nasal $\mathbf{n}$-, puis sur les diverses fonctions de $\mathbf{m}$ en positions initiale et finale de mot. Nous montrerons qu'on peut dériver ces consonnes nasales à partir des consonnes non nasales précédant une nasale moraïque / / 


\subsection{Le préfixe nasal $n-$}

43 La consonne [n] fonctionnecomme un préfixe verbal marquant une action en cours, comme dans les exemples en (11) ci-dessous.

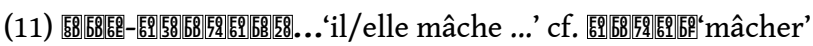

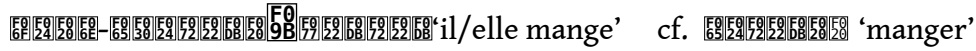

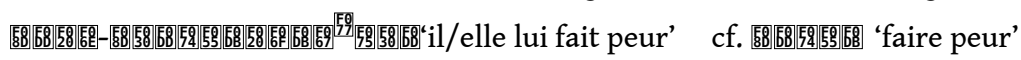

Dans ces exemples, la voyelle de l'infinitif qui suit [n] est nasalisée. Il convient cependant, de se rappeler qu'à l'exception de ce cas, les voyelles nasales n'apparaissent jamais en position initiale de mot, comme illustré par les formes à droite.

Etant donné toutes ces formes, on pourrait penser que l'on a affaire à une nasale sousjacente /監-/. Or nous avons vu dans d'autres contextes qu'un[n] phonétique est toujours dérivé d'un /1/ sous-jacent devant une voyelle nasale. On peut faire la même analyse pour

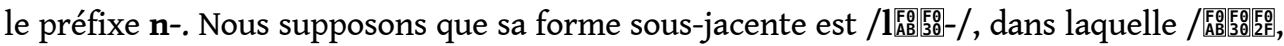
la nasale moraïque, n'est pas spécifiée pour un ton ou pour toute autre qualité vocalique. Cette nasale moraïque déclenche la nasalisation de /1/ en [n], puis subit une règle d'élision vocalique.

Cette dernière règle,d'une très grande généralité en ikwere, efface le premier membre d'une suite de deux voyelles avec le transfert de son trait [+nasal], si elle en a un, à la voyelle suivante.Considérons les exemples en (12):

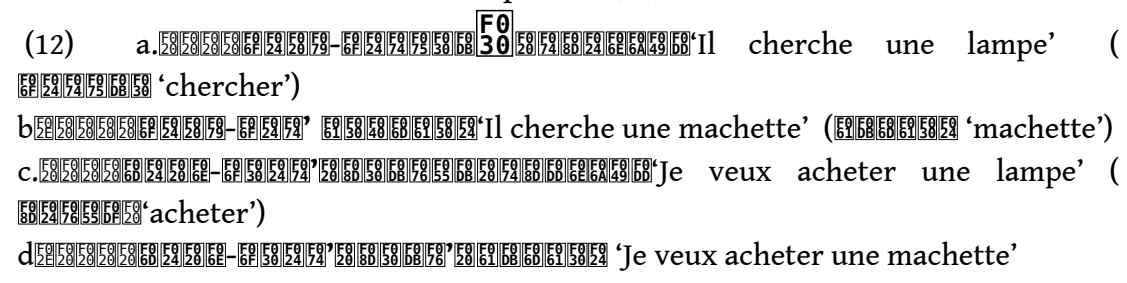

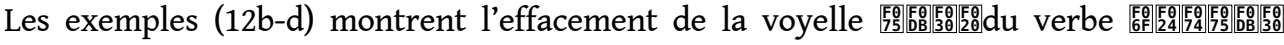
'chercher' devant un mot commençant par une voyelle. (L'exemple (12d) montre aussi l'effacement de la voyelle du verbe est nasale, son trait [+nasal] passe à la voyelle suivante, qui est normalement orale comme il est montré par les formes à l'infinitif à droite.

C'est ce même processus d'élision vocalique qui explique la nasalisation de la voyelle post-préfixale en (11); ainsi dans la troisième phrase, par exemple, on obtient la

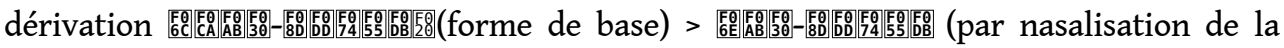

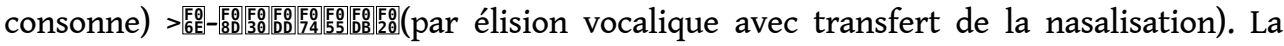
voyelle sous-jacente / / que devant la voyelle qui marque l'infinitif, ce qui entraîne automatiquement sa suppression.

L'analyse que nous proposons pour [n-] permet d'expliquer la nasalisation de la voyelle

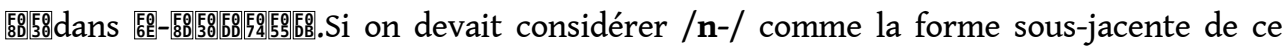
préfixe, on serait obligé de postuler un nouveau phonème qui n'apparaîtrait que dans ce préfixe. 


\section{2. [m] en position finale et le préfixe [m-]}

50 Considérons maintenant la consonne [m].En plus des contextes déjà examinés, $[\mathbf{m}]$ apparait 1) en position finale de mot et 2) en position initiale de mot, où il constitue la

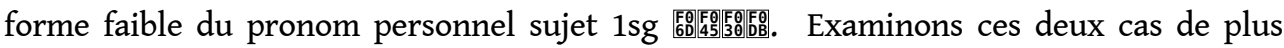
près.

51 Premièrement, [m] apparaît à la fin de certaines racines. La voyelle qui le précède peut être orale comme en (13a) ou nasale comme en (13b), bien que ce dernier cas de figure soit plus rare.

(13) a. la voyelle qui précède est orale:

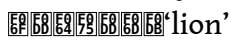

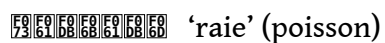

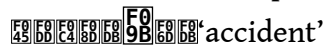

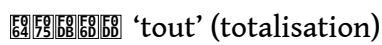

颉

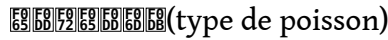

b. la voyelle qui précède est nasale:

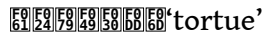

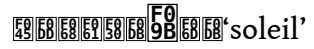

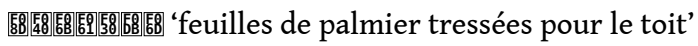

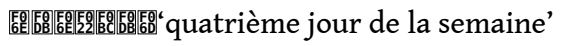

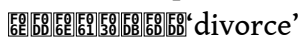

52 Comme le montrent ces exemples, le $\mathbf{m}$ en position finale peut ajouter un ton haut $(\mathrm{H})$, un ton bas (B) ou encore un ton haut abaissé $\left({ }_{\mathrm{gg}}^{\mathrm{E}} \mathrm{H}\right)$ au schème tonal. (Dans les mots où son ton est identique à celui de la voyelle qui le précède nous ne l'indiquons pas dans notre

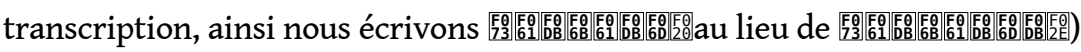

Le $\mathbf{m}$ final peut aussi représenter le suffixe de la $1^{\text {ère }}$ personne singulier possessif et objet (14a, 14b). Il est aussi la marque d'accord de la $1^{\text {ère }}$ personne singulier (14c).

(14) a. suffixe 1sg possessif :

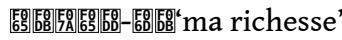

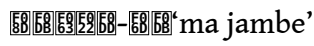

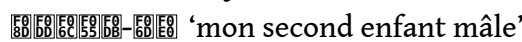

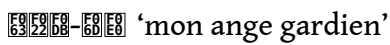

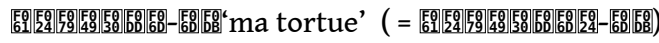

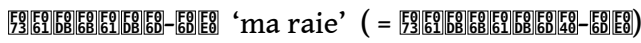

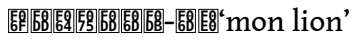

b. suffixe 1sg objet:

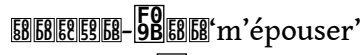

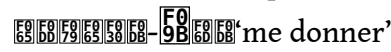

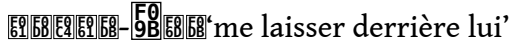

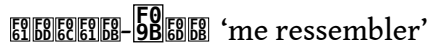

c. marque d'accord 1sg:

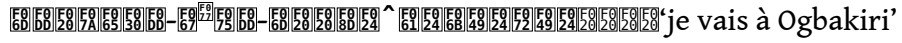

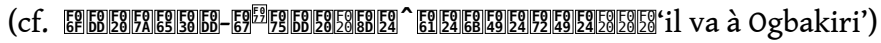

Dans le cas du suffixe 1sg possessif (14a), $\mathbf{m}$ porte un ton $\mathrm{H}$ si le ton qui le précède est $\mathrm{B}$ et un ton $\mathrm{BH}$ si le ton qui le précède est $\mathrm{H}$. Comme le montrent les trois derniers exemples, cette règle vaut également pour les bases qui se terminent par un $\mathbf{m}$. Dans le cas du suffixe 1sg objet (14b), m porte toujours un ton $\mathrm{H}$ abaissé. 

distinct $/ \mathbf{m}$ / étant donné que celui-ci n'est pas suivi en surface par une voyelle nasale susceptible de motiver sa dérivation à partir de $/ \AA$. Cependant, ce $\mathbf{m}$ aurait une distribution très incomplète : il n'apparaît qu'en position finale de mot où aucune autre

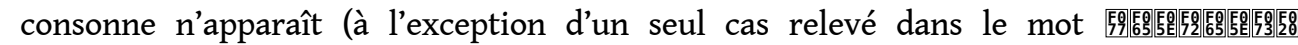
'pigeon'). En outre, on serait obligé d'admettre l'existence des séquences constituées

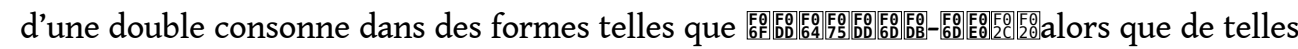
formes n'existent pas ailleurs en ikwere. Encore plus gênant est le fait qu'une telle analyse ne saurait expliquer pourquoi le $\mathbf{m}$ en position finale de mot porte un ton, alors que, normalement, seules les voyelles (y compris bien évidemment la nasale moraïque) portent un ton.

beaucoup de langues africaines, les nasales qui portent un ton sont dérivées historiquement et parfois synchroniquement des formes dans lesquelles la nasale précède une voyelle. Lorsque la voyelle dans une telle séquence est supprimée, le ton se

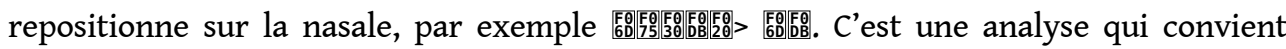
parfaitement au cas que nous étudions ici. Mais la question est la suivante : au niveau sous-jacent, quelle est la voyelle qui pourrait suivre le $\mathbf{m}$ en position finale en ikwere ? On peut penser qu'il s'agit d'une voyelle nasale étant donné qu'elle conditionne la variante nasalisée de la consonne. Cependant, comme la voyelle n'apparaît jamais en surface, ses autres traits sont indéterminés. En particulier, la voyelle ne peut être spécifiée comme [+ATR] ou [-ATR] puisque $\mathbf{m}$ peut apparaître dans les mots de l'une ou l'autre de ces deux catégories (voir les exemples en (13)).

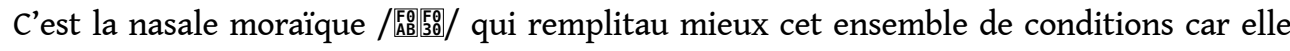
peut porter un ton et en même temps, n'a pas de lieu d'articulation spécifique (cf. section

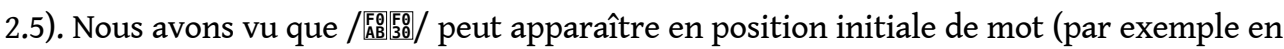

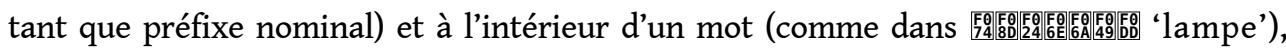
mais nous ne l'avons pas encore trouvée en position finale de mot. On peut expliquer cette lacune distributionnelle si l'on part de l'hypothèse qu'elle apparait au niveau sousjacent dans cette position après ^, mais qu'elle s'élide en surface. On pourrait alors

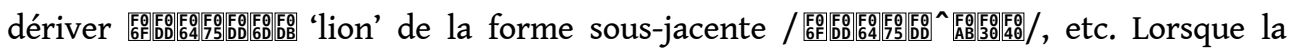
nasale moraïqueest supprimée en position finale, son ton et son trait [+nasal] s'associent au segment qui la précède, ce qui engendre une nasale $[\mathrm{m}]$ porteur de ton.

Cette analyse est étayée par plusieurs observations. D'abord, elle explique les caractéristiques particulières $\mathbf{d u} \mathbf{m}$ en position finale de mots tel que nous les avons résumées ci-dessus. En plus, elle rend possible une analyse unifiée des formes différentes que prend le pronom personnel $1 \mathrm{sg}$ : toutes peuvent être traitées comme des réductions

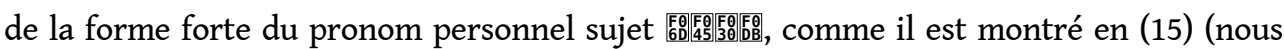
n'indiquons pas les tons).

(15) forme sous-jacente en surface

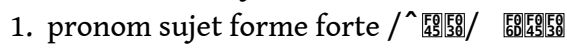

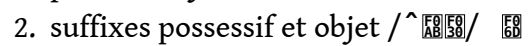

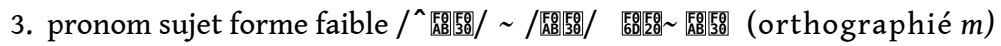

Selon cette analyse, la forme sous-jacente $/ /^{\wedge}$ Fin日: gauche) est identique à celle de la forme forte du pronom sujet (ligne 1), sauf que sa

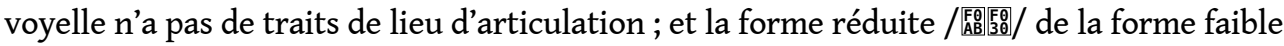
du pronom sujet (ligne 3) est également la même que la forme de la ligne 2 , à ceci près 
qu'il lui manque sa consonne initiale. On peut observer une érosion progressive des traits

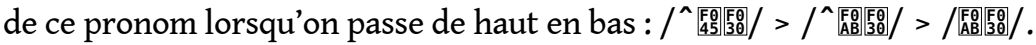

60 Un autre argument pour soutenir notre analyse $\mathbf{d u} \mathbf{m}$ en position finale de mot est d'ordre purement phonologique. Toute analyse qui dériverait des syllabes de la forme

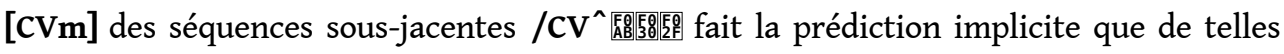
syllabes doivent présenter le même ensemble de schèmes tonals que l'on trouve dans les autres séquences $/ \mathrm{CVCV} /$,à ceci près que ces schèmes seront soumis aux contraintes qui gouvernent les suites de tons à l'intérieur d'une syllabe.

61 Cette prédiction est confirmée par les données, comme le montrent les formes en (16) cidessous.

(16) dissyllabes syllabes se terminant par $\mathbf{m}$

B B B

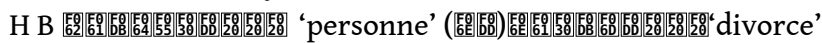

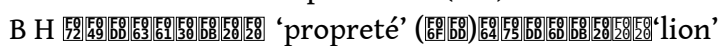

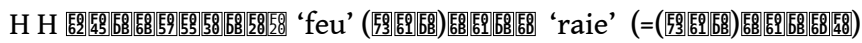

H 䜿H

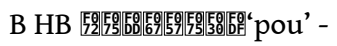

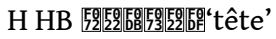

62 On peut constater à partir de la première colonne que les noms dissyllabiques peuvent porter un des sept schèmes tonals possibles en ikwere. Ces combinaisons de $\mathrm{H}$ et $\mathrm{B}$ sont soumises aux quatre contraintes suivantes: 1 ) aucune syllabe ne peut porter une suite $\mathrm{BH}$ (ton montant), 2) les tons descendants (HB) apparaissent seulement sur la syllabe finale, 3) les tons $\mathrm{H}$ abaissés n'apparaissent que sur la syllabe finale et seulement après un ton $\mathrm{H}$, et 4) aucune syllabe ne porte plus de deux tons'.

La seconde colonne montre que les syllabes finales qui se terminent par $\mathbf{m}$ présentent cinq de ces schèmes uniquement. Les deux schèmes manquants sont BHB et $\mathrm{HHB}$, ce qui est indiqué par des tirets dans les deux dernières lignes. On peut expliquer leur absence par la quatrième contrainte citée ci-dessus, qui interdit plus de deux tons sur une seule syllabe. En d'autres termes, les deux lacunes en (16) sont tout à fait prévisibles.

64 Enfin, la première colonne montre également que les syllabes finales en ikwere présentent seulement quatre schèmes contrastifs : $\mathrm{H}, \mathrm{B}$, descendant $(\mathrm{HB})$ et montant $(\mathrm{BH})$. Si les syllabes finales [CVm] étaient monosyllabiques au niveau sous-jacent, on s'attendrait à ce qu'elles ne portent que ces quatre schèmes. La présence d'un cinquième

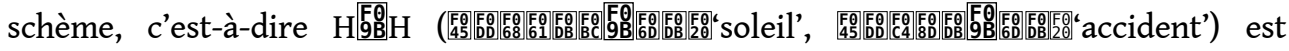
expliquée par leur origine dissyllabique ${ }^{10}$.

65 Ainsi, les propriétés phonologiques $\mathbf{d u} \mathbf{m}$ en position finale de mot et $\mathbf{d u} \mathbf{m}$ de la forme faible du pronom sujet 1 sg proviennent synchroniquement de celles des syllabes en $/{ }^{\wedge}$ 周国阳

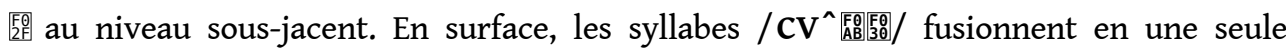
syllabe [CVm] dont $\mathbf{m}$ constitue une more portant son ton propre. Cette syllabation en surface, déjà corroborée par les différentes formes de neutralisation tonale décrites cidessus, est à nouveau soutenue par des faits relatifs à la réalisation phonétique. Dansla deuxième syllabe d'un mot comme

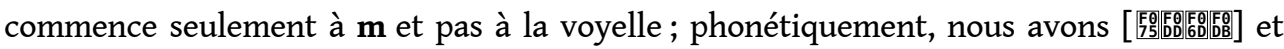

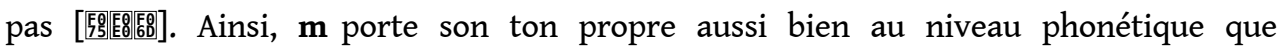
phonologique. Mais dans la mesure où la nasale moraïque fait partie d'une syllabe au lieu de former une syllabe à elle seule, son ton fait également partie de la mélodie tonale de la même syllabe. D'une manière générale, les syllabes à ton montant sont plus longues que 


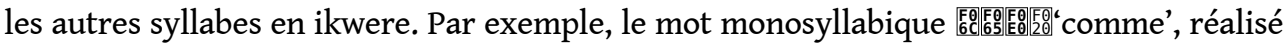

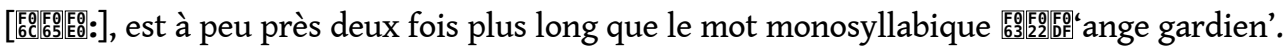
De même, la longueur des syllabes se terminant par $\mathbf{m}$ et qui portent un ton montant est deux fois celle des autres syllabes qui se terminent par $\mathbf{m}$. Par exemple, la syllabe finale

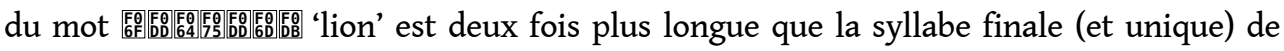

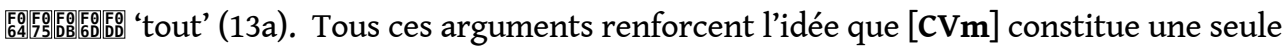
syllabe au niveau de la surface.

\section{En résumé}

Nous avons montré, données à l'appui, que la nasalité n'est pas un trait distinctif des consonnes en ikwere.Parmi les consonnes, les non-obstruantes se réalisent nasales devant les voyelles nasales mais orales devant les orales, tandis que les obstruantes se réalisent toujours orales. Comme nous l'avons vu, cette caractéristique se retrouve dans beaucoup d'autres langues de l'Afrique de l'Ouest.

Nous avons souligné que d'un point de vue phonologique, le processus de nasalisation en ikwere concerne une classe naturelle de sons (les non-obstruantes), et que d'un point de vue phonétique, les voyelles nasales qui suivent les consonnes nasales sont aussi fortement nasalisées que les voyelles nasales suivant les consonnes orales. Ainsi notre analyse n'est pas seulement justifiée sur la base d'une analyse distributionnelle formelle, comme dans les analyses phonémiques classiques, elle est aussi fondée sur des faits typologiques, phonologiques, et phonétiques. ${ }^{11}$

\section{BIBLIOGRAPHIE}

Beddor, Patrice S., Rena A. Krakow, \& Stephanie Lindemann (2001). Patterns of perceptual compensation and their phonological consequences. Dans Hume, E. \& Johnson, K. (éds.), The Role of Speech Perception in Phonology, San Diego : Academic Press. 55-78.

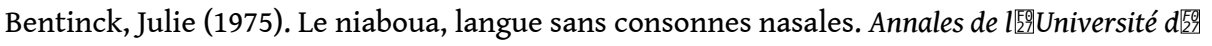
Abidjan, Série H, tome VIII. 5-14.

Bole-Richard, Rémy (1983a). Systématique phonologique et grammaticale d'un parler éwé : le gen-mina du sud-togo et sud-bénin. Paris: Éditions l'Harmattan.

Bole-Richard, Rémy (1983b). Ébrié. Dans Hérault, G. (éd.), Atlas des langues kwa de Côte d'Ivoire, Tome 1 : Monographies. Abidjan : ILA. 307-357.

Bole-Richard, Rémy (1984). Le ngwla, langue sans consonne nasale. Cahiers Ivoiriens de Recherche Linguistique (CIRL) 16. 23-35.

Bole-Richard, Rémy (1985). Hypothèse sur la genèse de la nasalité en Niger-Congo. Journal of West African Languages 15.2. 3-28.

Capo, Hounkpati B.C. (1981). Nasality in Gbe: a synchronic interpretation. Studies in African Linguistics 12. 1-43. 
Capo, Hounkpati B.C. (1991) A Comparative Phonology of Gbe.Berlin : Foris and Garome: Labo Gbe (Int).

Clements, G. N. \& Sylvester Osu (2002). Explosives, implosives, and nonexplosives: some linguistic effects of air pressure differences in stops. Dans Gussenhoven, C. \& Warner, N. (éds.), Laboratory Phonology 7. Berlin : Mouton de Gruyter. 299-350.

Clements, G. N. \& Sylvester Osu (2003). Ikwere nasal harmony in typological perspective. Dans Sauzet, P. \& Zribi-Hertz, A. (éds.), Typologie des langues d'Afrique et universaux de la grammaire, vol. 2. Paris : L'Harmattan. 70-95.

Clements, G. N. \& Sylvester Osu (2005). Nasal harmony in Ikwere, a language with no phonemic nasal consonants. Journal of African Languages and Linguistics 26.2.165-200.

Clements, G. N. \& Annie Rialland. (2008). Africa as a phonological area. In Bernd Heine \& Derek Nurse, eds, ALinguistic Geography of Africa, 36-85. Cambridge University Press.

Cohn, Abigail C. (1993a). The status of nasalized continuants. Dans Huffman, M. K. \& Krakow, R. A. (éds.), Phonetics and Phonology 5 : Nasals, Nasalization, and the Velum. N.Y. : Academic Press. 329-367.

Cohn, Abigail C. (1993b). A survey of the phonology of the feature [ \pm nasal]. Working Papers of the Cornell Phonetics Laboratory 8. 141-203.

Creissels, Denis (1994). Aperçu sur les structures phonologiques des langues négro-africaines, $2^{\mathrm{e}}$ édition. Grenoble : ELLUG, Université Stendhal.

Donwa-Ifode, S.O. \& S.A. Ekwulo (1987). Ikwere orthography. Dans Orthographies of Nigerian Languages : Manual (version remaniée par R.N. Agheyisi). Lagos : National Language Centre, Federal Ministry of Education

Ferguson, Charles A. (1963). Assumptions about nasals : a sample study in phonological universals. Dans Greenberg, J. H. (éd.), Universals of Language. Cambridge, MA. : MIT Press. 53-60.

Ferguson, Charles A. (1975). Universal tendencies and 'normal' nasality. Dans Ferguson, C.A., Hyman, L.M. \& Ohala, J. (éds.), Nasálfest : Papers from a Symposium on Nasals and Nasalization. Stanford : Department of Linguistics, Stanford University. 175-196.

Ihionu, Peter U. (1984). Nasality and Vowel Harmony in Igbo : an Autosegmental Approach. Unpublished M.A. dissertation, University of Ilorin.

Ladefoged, Peter, Kay Williamson, Ben Elugbe \& A. Uwulaka. (1976). The stops of Owerri Igbo. Studies in African Linguistics, Supplement 6.147-163.

Le Saout, Joseph. (1973). Langues sans consonnes nasales. Annales de l'Université d'Abidjan, Série H, Tome VI. 179-205.

Maddieson, Ian (1984). Patterns of sounds. Cambridge : Cambridge University Press.

Martinet, André (1955). Économie des changements phonétiques : traité de phonologie diachronique. Berne : Éditions A. Francke S.A..

Moñino, Yves (1995). Le proto-gbaya : essai d'application de la méthode comparative à un groupe de 21 langues oubangiennes. Paris : Peeters.

Ohala, John J. \& Manjari Ohala (1993). The phonetics of nasal phonology : theorems and data. Dans Huffmann, M.K. \& Krakow, R.A. (éds.), Phonetics and Phonology 5 : Nasals, Nasalization, and the Velum. N.Y. : Academic Press. 225-249. 
Osu, Sylvester (1998). Opérations énonciatives et problématique du repérage : cinq particules verbales

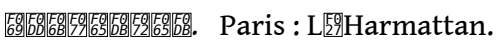

Schachter, Paul \& Victoria Fromkin (1968). A Phonology of Akan : Akuapem, Asante and Fante. (UCLA Working Papers in Phonetics 9). Los Angeles : University of California, Department of Linguistics.

Stevens, Kenneth N. (1983). Design features of speech sound systems. Dans MacNeilage, P.F. (éd.), The Production of Speech. New York : Springer-Verlag. 247-261.

Stewart, John M. (1989). Kwa. Dans Bendor-Samuel, J. (éd.), The Niger-Congo languages: a classification and description of Africa's largest language family. Lanham, New York, London: University Press of America. 217-245.

Stewart. John M. (1999). Compte-rendu de Moñino 1995, Journal of African Languages and Linguistics 20.1. 73-76.

Walker, Rachel. (1998). Nasalization, neutral segments, and opacity effects. PhD dissertation, University of California at Santa Cruz.

Williamson, Kay. (1970). Reading and writing Ikwerre ( $3^{e}$ impression 1980). Ibadan : Institute of African Studies.

Williamson, Kay. (1973). More on nasals and nasalization in Kwa. Studies in African Linguistics 4. 115-138.

Williamson, Kay. (2000). Reconstructing Proto-Igboid obstruents. Dans Carstens, V. \& Parkinson, F. (éds.). Trends in African Linguistics 4 (Proceedings of ACAL 28). Trenton, N.J. : Africa World Press. $1-18$.

Williamson, Kay \& Roger Blench. (2000). Niger-Congo. Dans Heine, B. \& Nurse, D. (éds.), African Languages : an Introduction. Cambridge : Cambridge University Press. 11-42.

Worukwo, Glory Ikechukwu (1983). The verbal system of Ikwere (based on the Ogbakiri dialect). B.A. long essay, University of Port Harcourt, Nigeria.

\section{NOTES}

1. Source : recensement 1991, publié dans the Annual Abstract of Statistics par the Federal Office of Statistics, Nigeria, 1998.

2. Pour ce qui est de l'étude de la nasalité dans d'autres langues igboïdes, on peut signaler Ihionu (1984). Il s'agit d'une étude approfondie de l'harmonie nasale et vocalique dans le parler igbo de Mbaise. Le système de ce parler igbo s'avère très proche de celui de l'ikwere.

3. Une étude phonétique montre que les mots avec une voyelle à l'initiale tels que ceux en (1) sont régulièrement initiés par une consonne occlusive glottale (Clements \& Osu 2002), et ceci afin peut-être de remplir une position d'attaque syllabique vide. La section 2.5 montre que l'élément nasal dans les séquences NC à l'initiale de mot est un élément syllabique (plus précisément, une voyelle nasale sans lieu d'articulation spécifié), tandis que la section 3.2 montre que les nasales en finale de mot peuvent être considérées au niveau sous-jacent comme des attaques des syllabes comportant une voyelle nasale.

4. Remarquons que ni une glottale [h] ou [?], ni le préfixe nasal moraïque ne déclenchent la nasalisation en ikwere.

5. Dans certains parlers ikwere, le mot 'joue', orthographié àgbnà, correspond ààbà̀(avec l'obstruante [b]) du parler d'ogbakiri et de beaucoup d'autres parlers (à signaler que certains parlers ont une autre variante encore àgwnà). Ce mot contient un son orthographié gb devant 
une voyelle nasale, une nasalité que l'on représente à l'aide de $n$ dans l'orthographe.Nous ne connaissons pas bien encore la nature phonétique de ce $g b$. S'agirait-il d'une obstruante labiovélaire comme le suggère le symbole $g b$ de l'orthographe, ce mot ne saurait constituer une exception à l'harmonie nasale, qui n'affecte que les non-obstruantes. Par ailleurs, si ce son remplaçait totalement [à] dans ce dialecte, [m] n'aurait aucune correspondante orale dans la série de non-obstruantes et elle serait dans ce cas, phonémique. Nous ne sommes pas en mesure d'en dire plus du fait du manque de travaux de description phonétique et phonologique des dialectes en question. Il est à noter cependant, que la distribution complémentaire des nonobstruantes orales et nasales que nous décrivons dans cet article est, en l'état actuel de notre connaissance de l'ikwere, valable pour la majorité des parlers de cette langue.

6. Bien que $\mathbf{m}$ apparaisse aussi en position finale du mot, comme il sera discuté en section 3.2, il n'est pas en contraste avec des consonnes orales dans ce contexte.

7. Les sons [m]et['m] se distinguent aussi de leurs correspondantes orales par le fait qu'ils sont caractérisés par le trait [+sonant], tandis que [n] se distingue de [1] par le fait qu'il lui manque le trait [+lateral].

8. Une autre analyse des faits de l'éwé est pourtant possible. En effet, les consonnes qui se nasalisent peuvent être traitées comme une classe naturelle s'il est admis que le trait [ \pm obstruant] est sous-spécifié pour cette classe, aussi bien en éwé qu'en ikwere. Dans cette analyse (Clements et Rialland 2008), la règle de nasalisation porterait sur l'ensemble de consonnes (une classe naturelle), mais une contrainte indépendante interdisant la combinaison de valeurs [+obstruant, +nasal] empêcherait la propagation de la nasalité sur les obstruantes.

9. Le deuxième ton $\mathrm{H}$ du schème $\mathrm{H} \mathrm{HL}$, illustré ici par rísî 'tête 匫, est toujours réalisé comme un ton $\mathrm{H}$ abaissé ; puisque cette réalisation est prévisible nous ne le transcrivons pas.

10. Une autre analyse de ces faits est possible, selon laquelle les syllabes [CVm] seraient de simples syllabes à deux mores, chacune porteuse de ton, aussi bien au niveau sous-jacent qu'en surface. La more nasale $[\mathbf{m}]$ serait une variante contextuelle, restreinte à la position finale de mot, de la more nasale /əح/. Le principal inconvénient de cette analyse est qu'en dehors de ces syllabes, l'ikwere ne possède que les syllabes simples /CV/. Notre analyse permet donc, de maintenir que l'ikwere est une langue CV sans exceptions.

11. Voir Clements \& Osu (2003) pour une présentation de l'harmonie de nasalisation en ikwere dans une perspective typologique.

\section{AUTEURS}

\section{SYLVESTER OSU}

Université François Rabelais, Tours/LLL-EA 3850, Orléans-Tours. sylvester.osu@wanadoo.fr

\section{G. N. CLEMENTS}

Laboratoire de Phonétique et Phonologie (UMR 7018) CNRS/Sorbonne-Nouvelle. nick.clements@univ-paris3.fr 